\title{
Perspectivas \\ Nutrición Humana
}

\author{
Escuela de Nutrición y Dietética \\ de la Universidad de Antioquia \\ Vol. $23, \mathrm{~N}^{\circ}{ }^{\circ}$, enero-junio de 2021
}

\section{Juan Carlos Santacruz ${ }^{1}$}

En Colombia, desde el 24 de marzo hasta el 31 de agosto de 2020, sus pobladores debieron enfrentar el reto de 162 días en aislamiento preventivo obligatorio por la pandemia de la COVID-19. La Fundación Colombiana del Corazón aplicó dos encuestas virtuales abiertas, la primera para evaluar el impacto del confinamiento en la actividad física diaria y el peso de los niños y la segunda para dimensionar el significado de la naturaleza en el bienestar de las personas y su percepción frente a la ausencia del contacto con la naturaleza.

\section{Impacto en la actividad física diaria y en el peso de los niños}

Un total de 1139 encuestas virtuales permitieron establecer que durante el periodo de aislamiento obligatorio el $75,2 \%$ de los niños no realizó los minutos recomendados de actividad física por la Organización Mundial de la Salud (OMS), que el $82,8 \%$ superó el tiempo recomendado de 60 minutos frente a dispositivos electrónicos y que el $44 \%$ de los niños aumentó de peso (1).

La medida de aislamiento obligatorio era inédita en el país, nunca lo había afrontado la población colombiana, y en cuanto los niños, específicamente, ese aislamiento empezó a provocar diversas polémicas sobre si era necesario y si fue bien argumentado técnicamente. La evidencia disponible aún es muy poca para sacar conclusiones, sobre todo porque los estudios del impacto del aislamiento en los niños deben evaluar consideraciones sociales, económicas e incluso epidemiológicas. Durante las 20 semanas de aislamiento obligatorio del 2020, surgió una fuerte polémica sobre el 
impacto que esta situación obligada tendría sobre la salud física y mental de los niños, por las restricciones impuestas en la práctica de la actividad física, la ausencia de contacto con la naturaleza y la imposibilidad de realizar juegos al aire libre (2).

Los resultados obtenidos en la primera encuesta aplicada por la Fundación Colombiana del Corazón fueron claros en el evidente incremento de las actividades sedentarias y el decrecimiento de la actividad física en los niños, como consecuencia del confinamiento. Estos resultados y su interpretación se convierten en un insumo para continuar los estudios e investigaciones sobre las consecuencias de las determinaciones gubernamentales ante la llegada del nuevo coronavirus COVID-19 en la población de niños y adolescentes en Colombia.

La niñez y la adolescencia son periodos críticos para el desarrollo de habilidades motoras y para aprender conductas saludables orientadas a establecer la base de la salud del futuro adulto, así como la perfección del bienestar asociado con estilos de vida que prioricen comportamientos orientados al cuidado. El aislamiento puede traer como consecuencia niños más débiles, más lentos y pesados en comparación con ellos mismos antes de iniciarse la coyuntura sanitaria; además, se evidenciará lo que se conoce como la tríada de inactividad pediátrica (PIT) con trastorno de déficit de ejercicio, dinapenia pediátrica y analfabetismo físico (3).

\section{Percepción del significado de la ausencia de contacto con la naturaleza}

En el segundo estudio realizado por la Fundación Colombiana del Corazón, se entrevistó un total de 1849 personas en Colombia, lo que permitió establecer que una de cada cinco personas nunca tuvo contacto con la naturaleza durante la cuarentena, que el 91,4 \% la planteó como una necesidad, el 98,6 \% precisó que la naturaleza aporta equilibrio físico y mental y el 96,1\% deseaba conocer cómo beneficiarse de la naturaleza.

La cuarentena impuso el confinamiento y el distanciamiento social, entre otros, como medidas indispensables para disminuir el riesgo de contagio. Sin embargo, también significó afectaciones sociales, psicológicas y económicas para la población. Así mismo, la disminución del contacto con la naturaleza y del cierre total de las opciones de cualquier tipo de actividad asociada con estar al aire libre, limitándose incluso las posibilidades de visitar parques urbanos en la misma ciudad.

Para los admiradores de la naturaleza, quienes de forma habitual practican el senderismo, la visitas a la montaña o las salidas al mar, significó ayuno total de naturaleza. Teniendo en cuenta los numerosos estudios que se han realizado en los últimos años sobre los beneficios del contacto consciente con la naturaleza, que han evidenciado su impacto en el bienestar individual y social, es de esperar que el aislamiento tenga efectos negativos tanto en el individuo como en su grupo social.

Entre los múltiples beneficios del contacto con la naturaleza para el bienestar humano se ha investigado que puede disminuir la presión arterial, la variabilidad de la frecuencia cardíaca, 
la tensión muscular y la liberación de las hormonas del estrés, como el cortisol y la adrenalina, $y$, en cambio, se estimulan las hormonas de la felicidad, como endorfinas, serotoninas, dopaminas y oxitocina (4).

Especialistas en psicología ambiental explican que el confinamiento trajo consigo una pobreza de estímulos. "Tenemos menos estímulos y menos variados. Desde la psicología ambiental esto se conoce como saciedad psíquica, esa sensación de redundancia, de que te cansas antes de todo, de que todo es más de lo mismo" (5, p. 2). La falta de estímulos de relación social podemos complementarla con la relación virtual, pero el tema sensorial es más difícil de suplir. En términos psicológicos, la cuarentena provocó en las personas depresión, estrés, nivel de ánimo bajo, irritabilidad, insomnio, síntomas de estrés postraumático y ansiedad. Aunque parezca una contradicción, el confinamiento acercó, o al menos generó, la reflexión en los ciudadanos sobre la importancia de valorar su relación con la naturaleza, de acuerdo con los resultados obtenidos por esta investigación.

Se hace indispensable diseñar estrategias pedagógicas y formativas para que las personas aprendan a disfrutar de los beneficios de la naturaleza con decisiones sencillas como parte de un descubrimiento, incluso generado por la misma cuarentena obligatoria; por ejemplo, cuidar plantas en casa, hacer pequeñas plantaciones de aromáticas en materos o simplemente disponerse a escuchar los sonidos de la naturaleza -cuyas frecuencias inciden en el equilibrio emocional, la gestión del estrés y la vitalidad-, hasta se puede poner como fondo de pantalla paisajes de montañas o playas como un estímulo para proyectar dónde se quiere estar.

La aplicación de la encuesta sobre naturaleza en cuarentena permitió establecer, de manera confiable - gracias a la muestra construida y especialmente a los resultados tan contundentes en sus porcentajes-, que se hace indispensable generar políticas públicas orientadas a educar en la asociación entre naturaleza y salud/bienestar, para que ese logro formativo pueda revertirse en mejores decisiones de las personas en su relación con el entorno natural, para hacer posible ese disfrute dentro de una condición de respeto y conservación del ambiente.

Dichas decisiones están relacionadas con las actividades al aire libre que los encuestados buscan realizar, lo cual denota una necesidad de articular políticas públicas de turismo, recreación, salud y conservación, que permitan a los ciudadanos realizar actividades como el senderismo, la observación de fauna y flora y la contemplación de la naturaleza.

En términos turísticos, las experiencias deben vincular el bienestar con la conservación de la naturaleza; en la recreación, ofrecer discursos que denoten la importancia de las actividades al aire libre y en contacto con la naturaleza; en lo relacionado con las políticas de salud, la prevención de la enfermedad debe cobrar mayor importancia en la gestión de la salud pública e incluir la naturaleza como activo vivo e indispensable para la vida humana; $y$ en términos de conservación, continuar promoviendo mensajes asociados a la relación inherente entre espacios verdes y bienestar para la humanidad. También se debe valorar el aporte de la naturaleza más próxima desde el contexto del hogar, de manera que las personas construyan relaciones 


\section{Hierro y alimentación complementaria}

fructíferas con los otros seres vivos como plantas y animales y con los entornos naturales de veredas, barrios y ciudades.

Los resultados de este estudio son el punto de partida para fortalecer todos los procesos de educación en salud, para que los pobladores puedan beneficiarse de un estilo de vida que haga posible sumar minutos diarios de contacto con la naturaleza y se logre impactar de manera positiva en la salud física, mental, emocional y social de las personas, pues la necesidad de estar en contacto directo con la naturaleza tomó fuerza durante la cuarentena decretada a causa de la COVID-19 y hoy los individuos son más conscientes de lo que significa en su vida cotidiana.

\section{-Referencias}

1. Arévalo $\mathrm{H}$, Urina-Triana M, Santacruz JC. Impacto del aislamiento preventivo obligatorio en la actividad física diaria y en el peso de los niños durante la pandemia por SARS-CoV-2. Revista Colombiana de Cardiología. 2020;27(6):589-96. https://doi.org/10.1016/j.rccar.2020.09.003

2. Human Rights Watch. Devastador impacto del COVID-19 para niños y niñas | Human Rights Watch [Internet]. Human Rights Watch. 2020 [Citado el 9 de julio de 2020]. Disponible en: https://www.hrw.org/es/news/2020/04/09/devastador-impacto-del-covid-19-para-ninos-y-ninas

3. Faigenbaum AD, Rebullido TR, MacDonald JP. Pediatric inactivity triad: A Risky PIT. Curr Sports Med Rep. 2018;17(2):45-7. https://doi.org/10.1249/JSR.0000000000000450

4. Capaldi C, Dopko R, Zelenski J. The relationship between nature connectedness and happiness: A meta-analysis. Frontiers in Psychology. 2014;5(976):1-15. https://doi.org/10.3389/fpsyg.2014.00976

5. Espino A. Hace falta una revolución eco-cultural para darnos cuenta de que no somos los dueños de la naturaleza. (2018, 11 de octubre). Revista Circle. Disponible en: https://www.revistacircle.com/2018/10/11/jose-antonio-corraliza-revolucion-eco-cultural-no-somos-duenosnaturaleza/ 